\title{
Prosthetic Knee Joint Infection by Rare Atypical Mycobacterium Fortuitum
}

\author{
Naqvi ZG* \\ Department of Orthopedics and Surgery, Chiniot General Hospital, Karachi, Pakistan
}

*Corresponding author: Naqvi ZG, Department of Orthopedics and Surgery, Chiniot General Hospital, Karachi, Pakistan, Tel: +923462757529, E-mail: zohaib.naqvi1@gmail.com

Citation: Naqvi ZG (2018) Prosthetic Knee Joint Infection by Rare Atypical Mycobacterium Fortuitum. Saj Case Rep 5: 408

Article history: Received: 01 December 2017, Accepted: 27 September 2018, Published: 28 September 2018

\begin{abstract}
Wound Infections are indeed common after surgeries, but becomes catastrophic if following surgeries like joint arthroplasties, where they are called as Prosthetic Joint Infection (PJIs), which may result in removal of the implant, sometime salvage or even loss of the limb. Mycobacterium tuberculosis in post total knee replacement is known entity but prosthetic joint infection with atypical mycobacterium is rare. Up to the date only few cases has been reported in the literature in respect to atypical mycobacterium fortuitum prosthetic joint infection. Following case is reporting a prosthetic knee joint infection in elderly lady with atypical Mycobacterium fortuitum not responded to recommended treatment and results in loss of the limb.
\end{abstract}

Keywords: Prosthetic Joint Infection (PJI); Non-Pigmented Rapidly Growing Mycobacteria (NPRGM); Surgical Site Infection (SSI)

\section{Case Presentation}

Our patient was 70 years lady with no known comorbid, presented to elsewhere hospital with signs and symptoms of chronic arthritis bilateral knee joints but more on the right side and underwent uneventful right total knee replacement in an elsewhere hospital. About a year later of the index surgery she presented to us with the complaint was swelling, tenderness, chronic draining sinuses scar and one active draining sinus, discharging serous discharge in popliteal region Figure 1.

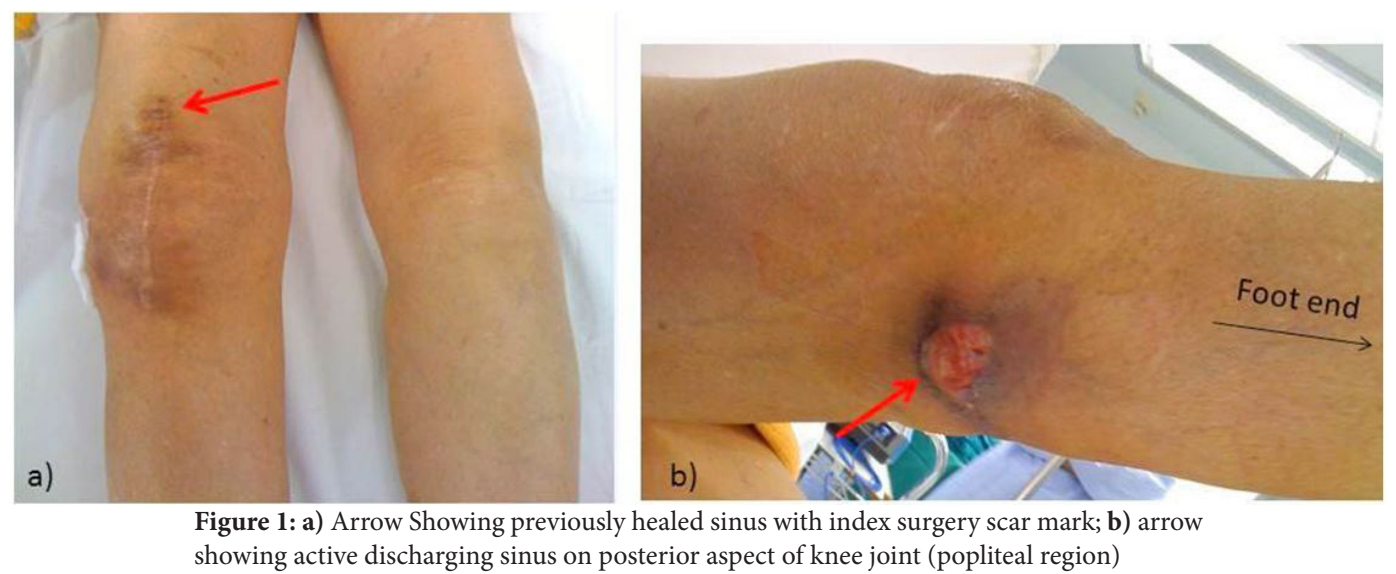

Laboratory investigations reveal normal Total leucocytes count (TLC): 15,000 ( $N=72.2$ ), increase C- reactive protein (CRP): 80 $\mathrm{mg} / \mathrm{dl}$ and increase Erythrocyte Sedimentation rate (ESR): $66 \mathrm{~mm} /$ hour. Diagnostic Arthrocentesis was done, synovial fluid detail report showed TLC; $22000 / \mathrm{cu} \mathrm{mm}$, polymorph: $98 \%$, lymphocytes: $2 \%$, glucose: $19 \mathrm{mg} / \mathrm{dl}$ that is non-specific and Synovial fluid Culture and sensitivity showed no growth. X-RAY knee at that time showed well fixed Implant with mild lucent area also appreciated in zone 1 and a normal chest X-RAY as in Figure 4 (Figure 2). Clinical impression was PROSTHETIC JOINT INFECTION. She underwent right knee debridement per operative findings reveals abundant granulation tissue, with no frank purulent fluid indicating that it's not pyogenic origin and well-fixed femoral and tibial components. She underwent right knee debridement and deep cultures were taken from multiple sites and were sent for routine smear and culture, Acid Fast Bacilli (AFB). Smear and culture, fungal smear and culture were sent along with the Histopathology and wound was closed primarily. Culture reveals numerous AFB on Ziehl- Neelsen Stain (both synovial and tissue). Histopathology showed chronic inflammatory cell with no granuloma or malignant cell seen. 6 weeks later AFB culture reveals atypical mycobacterium (fortuitum). 

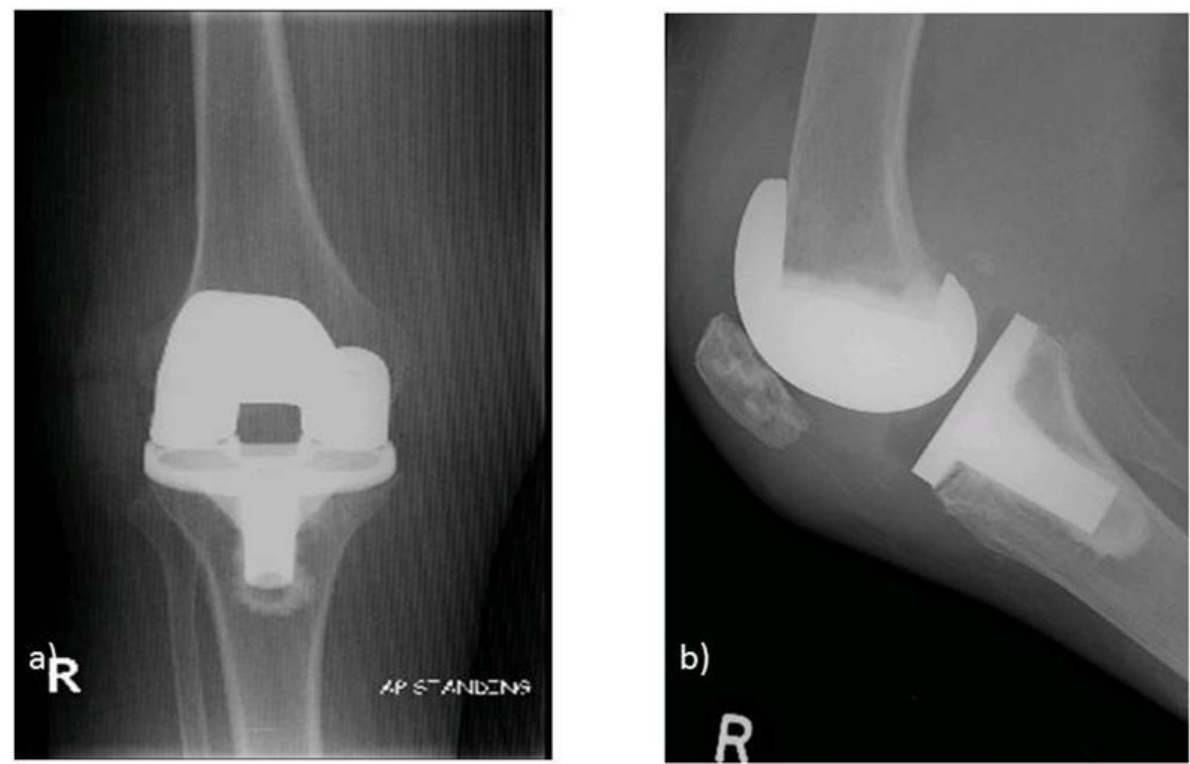

Figure 2: X-ray right knee showing well fixed implant in a) AP and b) Lateral views
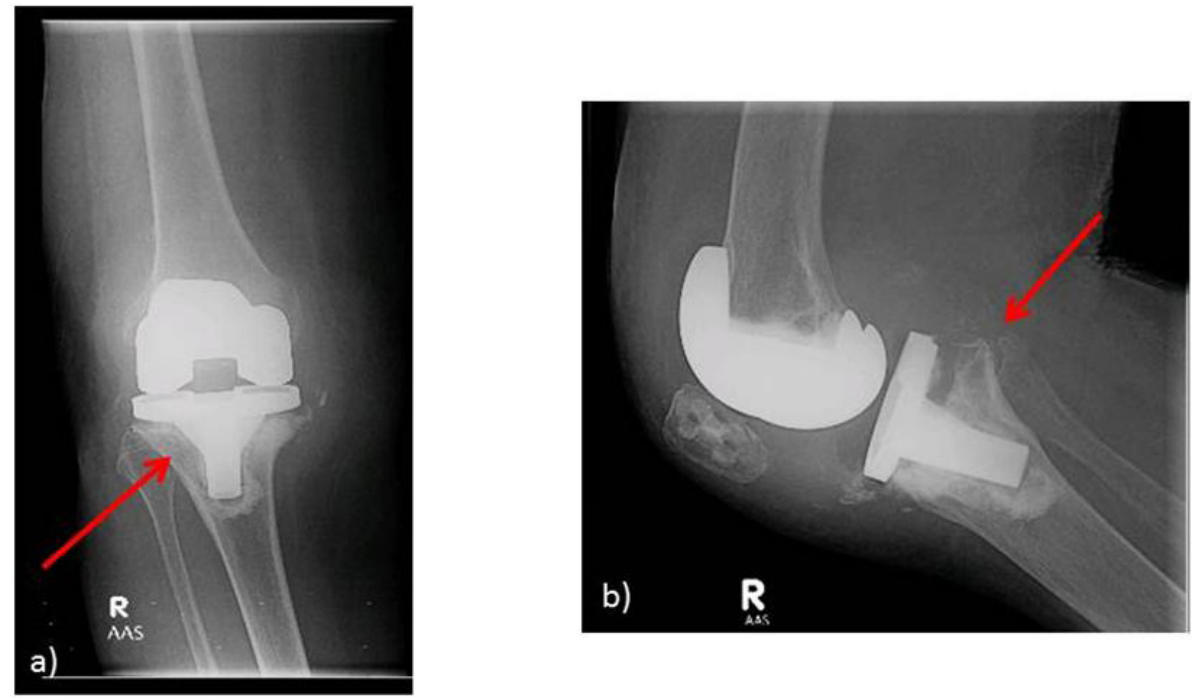

Figure 3: X-ray right knee, arrow showing global loosening of implant in a) AP and b) Lateral

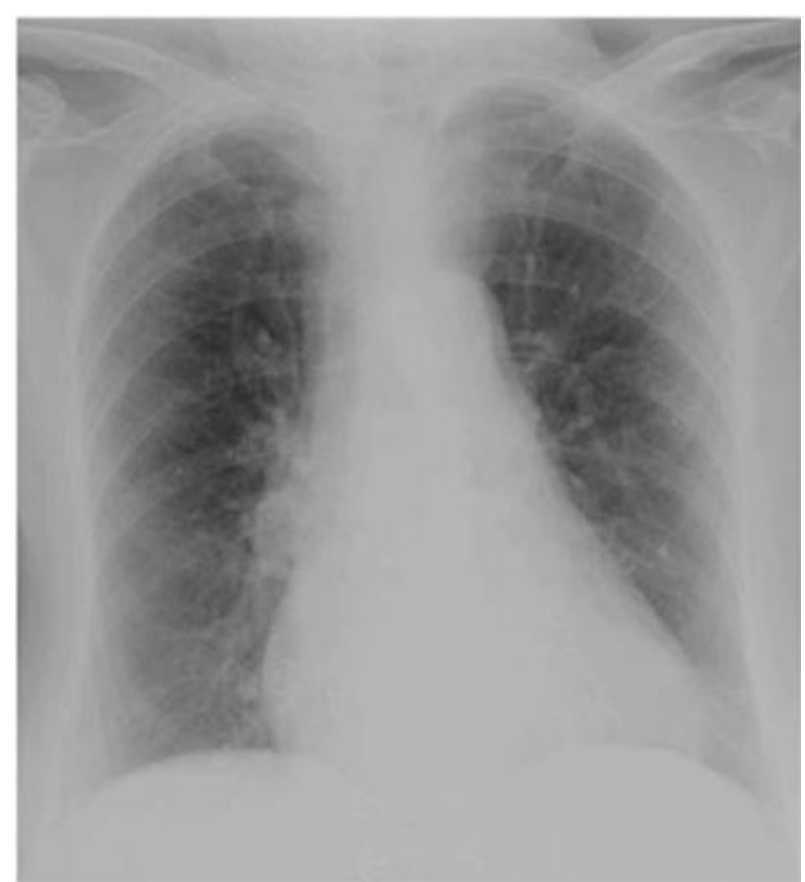

Figure 4: Showing normal chest X-ray 
She was started on recommended antibiotic (Clarithromycin and Doxycycline) and within six weeks of therapy there was some clinical remission, with resolution of pain and swelling, decreasing of sinus discharge, and some restoration of knee joint function. But following the treatment course patient became febrile, cachectic, worsening in general condition, developed exquisitely painful, swollen and stiffed knee with new site of discharging sinus. Labs showed TLC: 18,000 (N=72.2), increase CRP: 50.2 $\mathrm{mg} / \mathrm{dl}$ and increase ESR: $75 \mathrm{~mm} / \mathrm{hr}$. X-RAY right knee showed global loosening of both the components (tibial and femoral component). Per operative findings again reveals abundant granulation tissue, with no frank purulent fluid but loose femoral and tibial components as shown in X-RAYs and poor quality of bone. Patient underwent second debridement with the removal of implant and antibiotic spacer was placed despite of local and systemic antibiotic she did not showed remission in disease activity, then option was discussed with the patient and family regarding salvaging the limb via knee arthrodesis or above knee amputation (Figure 3). After detail discussion with the patient and the family, patient opted for above knee amputation in viewing to terminate the process of chronic suffering. On subsequent follow up patient showed good healing of the stump, normalization of laboratory parameters (ESR, C-reactive protein), and improvement in general state of health.

\section{Discussion}

Peri-prosthetic infection with Gram-positive organisms such as Staphylococcus aureus and Staphylococcus epidermidis are fairly common entity [1]. Despite of these routine encountered organisms, PJIs with mycobacterium tuberculosis also well known in our part of the world but PJI with Non- tuberculosis mycobacterium has been rarely seen and reported across the globe. This Nontuberculosis mycobacterium also called as Non-pigmented rapidly growing mycobacteria (NPRGM) are rare cause of prosthetic knee infection with unknown incidence [2-4]. It encompasses all mycobacterium outside of the mycobacterium complex, classified is classified in the Runyon group IV, a very rapidly growing mycobacterium. These types include Mycobacterium fortuitum, Mycobacterium goodii, Mycobacterium abscessus etc. [4,5]. These organisms are also uncommon for lung infection, but reported to occur as cutaneous disease and ocular diseases. On other hand SSI due to atypical mycobacterium is well documented especially in cardiothoracic surgery, but sparsely reported causing prosthetic knee joint infection (PJI) [6]. There rapidly growing characteristics also causing them resistant to the empirical anti-TTB drugs. Differential Diagnosis includes SSI, chronic prosthetic infection, tuberculosis or other mycobacterium infection. Diagnosis is usually based on chronicity of symptom and of course tissue biopsy and cultures are mandatory. Literature reveals successful treatment with multiple debridement's, removal of prosthesis and prolongs antibiotics esp. doxycycline and clarithromycin, but scenario changes in case of compromised host, resistant species and delay in diagnosis [7-10].

\section{Conclusion}

Prosthetic knee joint infection by atypical mycobacterium fortuitum is a rare infection which is difficult to diagnose and treatment. Despite the permutation of drugs due to atypical responses to medical treatment, patient ends up in sacrificing the limb.

\section{References}

1. Zimmerli W, Trampuz A, Ochsner PE (2004) prosthetic-joint infections. N Engl J Med 351: 1645-54.

2. Wang SX1, Yang CJ, Chen YC, Lay CJ, Tsai CC (2011) Septic arthritis caused by Mycobacterium fortuitum and Mycobacterium abscessus in a prosthetic knee joint: case report and review of literature. Intern Med 50: 2227-32.

3. Joseph MF (2015) Mycobacterium fortuitum. Drugs Dis.

4. Jaime Esteban, NZM-d-H, Teemu J Kinnari, Guillermo Ayala, Ricardo Fernández-Roblas et al. (2008) Biofilm development by potentially pathogenic non-pigmented rapidly growing mycobacteria. BMC Microbiol 8: 184

5. Yung Yu-Bun, LP-H, Lee Qunn-Jid, Wong Yiu-Chung, Wai Yuk-Leung (2012) Treatment of Mycobacterium fortuitum Infection of Total Knee Arthroplasty: A Case Report. J Orthopedic Trauma Rehabil 16: 82-5.

6. Lahiri K, Jena J Pannicker K (2009) Mycobacterium fortuitum infections in surgical wounds. Medi J Armed Forces India 65: 91-2.

7. Heathcock R, Dave J, Yates MD (1994) Mycobacterium chelonae hip infection. J Infect 28: 104.

8. Pring BA, Eckhoff DG (1996) Mycobacterium chelonae infection following a total knee arthroplasty. J Arthroplasty 11: 115-6.

9. Cuckler JM (2005) The infected total knee: management options. J Arthroplasty 20: 33-6.

10. Leone JM, Hanssen AD (2006) Management of infection at the site of a total knee arthroplasty. Instr Course Lect 55: 449-61. 